\title{
Eyebrow Regrowth in Patients with Frontal Fibrosing Alopecia Treated with Low-Dose Oral Minoxidil
}

\section{Rodrigo Pirmez ${ }^{a}$ Leonardo Spagnol Abraham ${ }^{b}$}

aDepartment of Dermatology, Instituto de Dermatologia Professor Rubem David Azulay, Santa Casa da Misericórdia

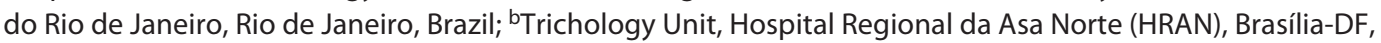
Brasília, Brazil

\section{Established Facts}

- Partial or complete eyebrow loss is seen in most patients with frontal fibrosing alopecia (FFA).

- Treatment options for eyebrow loss in FFA and supporting evidence remain scarce.

\section{Novel Insights}

- We report eyebrow regrowth in patients with frontal fibrosing alopecia (FFA) treated with low-dose oral minoxidil (OM).

- Low-dose OM could be a promising adjunctive therapy for treatment of the eyebrows in patients with FFA.

\section{Keywords}

Minoxidil · Frontal fibrosing alopecia · Cicatricial alopecia · Eyebrow

\begin{abstract}
Introduction: The eyebrows are an important facial feature that shape one's physical appearance and play a role in nonverbal communication. Partial or complete eyebrow loss is seen in most patients with frontal fibrosing alopecia (FFA). Despite the scarring nature of FFA, eyebrow hair regrowth has been previously reported. Nevertheless, treatment options and supporting evidence remain scarce. Case Presentation: We report eyebrow regrowth in 7 patients with FFA
\end{abstract}

treated with low-dose oral minoxidil (OM). Conclusion: Lowdose OM could be a promising adjunctive therapy for treatment of the eyebrows in patients with FFA, particularly in early disease.

() 2020 S. Karger AG, Basel

\section{Introduction}

The eyebrows are an important facial feature that shape one's physical appearance and play a role in nonverbal communication. Partial or complete eyebrow loss is seen in most patients with frontal fibrosing alopecia (FFA). It is uncommon for patients with FFA to 

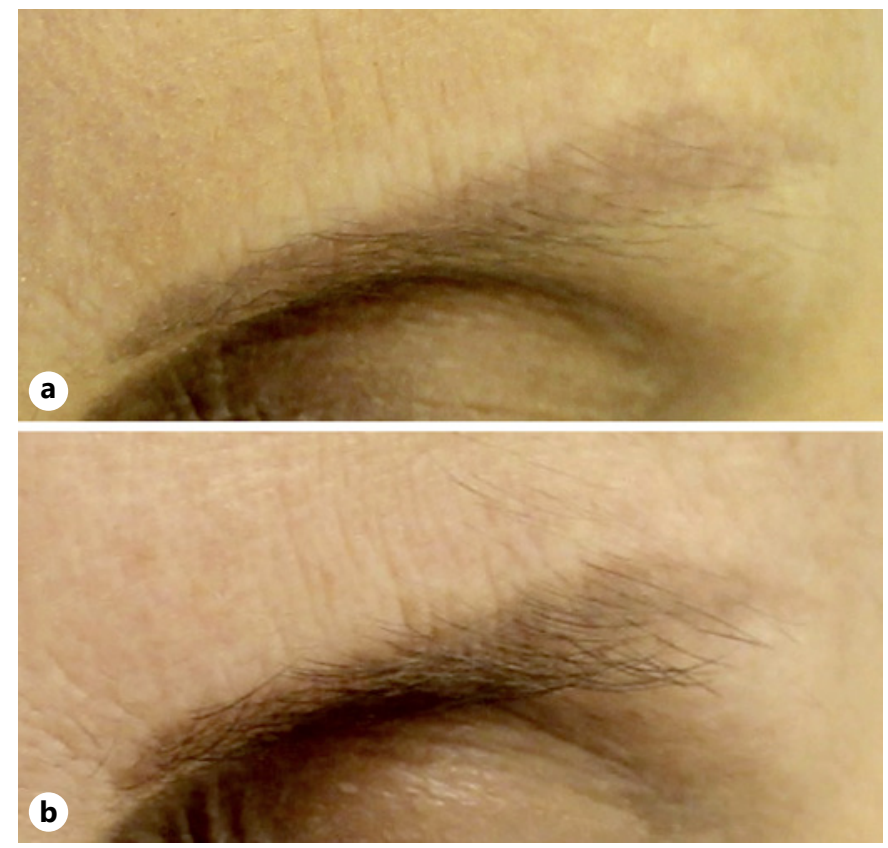

Fig. 1. Eyebrow regrowth in FFA. Picture from a 50-year-old patient with FFA before (a) and after (b) three-month-treatment with OM, showing partial regrowth of eyebrows. Eyebrow tattoo remained unchanged during the course of treatment. FFA, frontal fibrosing alopecia; OM, oral minoxidil.

experience eyebrow regrowth with systemic therapy alone [1]. Regrowth has been previously reported with intralesional triamcinolone and topical bimatoprost [1, $2]$, but treatment options and supporting evidence remain limited.

Recently, low-dose oral minoxidil (OM) has been successfully used for androgenetic alopecia (AGA) in both men and women $[3,4]$. The authors have used OM to increase hair volume in FFA patients with concomitant AGA. Interestingly, either partial or complete eyebrow regrowth was noted in a small cohort of patients after OM initiation.

\section{Case Reports}

We report 7 female patients with biopsy-confirmed FFA, age range 35-65 years, presenting with moderate eyebrow loss (less than $50 \%$ loss) who were treated with OM. The initial daily OM doses were $0.5 \mathrm{mg}$ ( 2 patients), $0.75 \mathrm{mg}$ ( 2 patients), and $1.25 \mathrm{mg}$ (3 patients). At month 3 , the dose was increased to $2.5 \mathrm{mg} /$ day in 5 patients, while 2 remained with $1.25 \mathrm{mg} /$ day. After 6 months, comparison of pre- and posttreatment pictures revealed eyebrow regrowth that was rated as partial in 5 and almost complete in 2 patients (shown in Fig. 1). Importantly, in all cases, regrowth was regarded by both physician and patient as cosmetically acceptable new eyebrow hair coverage. Of note, topical medications and intralesional steroids to the eyebrows were not used in any of the above patients. No serious adverse events were reported. In our experience, hypertrichosis secondary to $\mathrm{OM}$ is not as common in FFA compared to patients with AGA alone, possibly due to body hair involvement in the setting of FFA. However, interestingly, 1 patient reported regrowth of body hair in areas previously affected by the disease.

\section{Discussion}

Despite FFA's scarring nature on the scalp, eyebrow regrowth has been reported and is believed to be possible particularly in early cases of the disease. Indeed, trichoscopic features described for the eyebrows in FFA patients are mainly non-cicatricial [5]. The reason why hair loss at the eyebrows is, at least initially, non-cicatricial has not been fully investigated. Recently, Katoulis et al. [6] reported that sebaceous glands were preserved in eyebrow biopsies in $38 \%$ of FFA patients. Considering that sebaceous gland destruction is one of the earliest signs of cicatricial alopecia, its preservation could correlate with the reversibility of eyebrow loss observed in some cases [6].

Donovan et al. previously reported eyebrow regrowth in patients treated with intralesional triamcinolone acetonide at a concentration of $10 \mathrm{mg} / \mathrm{mL}$ [1]. However, continuous injections might be required in order to sustain treatment response, and skin atrophy has been reported even when lower concentrations such as $2.5 \mathrm{mg} / \mathrm{mL}$ were used [1, 7]. Recently, Murad and Bergfeld [2] reported eyebrow regrowth in a single patient treated with topical bimatoprost solution $0.03 \%$, which is a synthetic prostaglandin analog approved for hypothrichosis of the eyelashes.

The use of OM in FFA has not been explored. Anagen phase promotion of remaining viable hair follicles may account for the regrowth of eyebrow hairs in our patients. Additionally, it was recently suggested that minoxidil may also have anti-inflammatory properties via downregulation of interleukin-1-alpha expression [8]. We believe low-dose OM could be a promising adjunctive therapy for treatment of the eyebrows in patients with FFA, particularly in early disease. Larger randomized controlled trials are necessary not only to confirm our findings but to determine the best dosing regimen as well as verify the Number Needed to Treat of OM in FFA. 


\section{Statement of Ethics}

All patients gave written consent for publication including images, and research was conducted ethically in accordance with the World Medical Association Declaration of Helsinki.

\section{Conflict of Interest Statement}

The authors have no conflicts of interest to declare.

\section{Funding Sources}

There were no funding sources for this work.

\section{Author Contributions}

Every author listed meets the qualifications for authorship and has had the opportunity to read and comment upon the submitted manuscript. All authors listed provided substantial contributions to the conception of the work and interpretation of data. All authors participated in drafting the manuscript and revising it critically for important intellectual content. All authors approved the final version of the manuscript.

\section{References}

1 Donovan JC, Samrao A, Ruben BS, Price VH Eyebrow regrowth in patients with frontal fibrosing alopecia treated with intralesional triamcinolone acetonide. Br J Dermatol. 2010; 163(5):1142-4.

2 Murad A, Bergfeld WF. Prostaglandin analogue for eyebrow loss in frontal fibrosing alopecia: a case report. J Eur Acad Dermatol Venereol. 2019 May 22.

3 Pirmez R, Salas-Callo CI. Very low dose oral minoxidil in male androgenetic alopecia: a study with quantitative trichoscopic documentation. J Am Acad Dermatol. 2020 Jan; 82(1):e21-2.
4 Sinclair RD. Female pattern hair loss: a pilot study investigating combination therapy with low-dose oral minoxidil and spironolactone. Int J Dermatol. 2018;57(1):104-9.

5 Anzai A, Pirmez R, Vincenzi C, Fabbrocini G, Romiti R, Tosti A. Trichoscopy findings of frontal fibrosing alopecia on the eyebrows: a study of 151 cases. J Am Acad Dermatol. 2019 Dec 17.
6 Katoulis AC, Damaskou V, Diamanti K, Pouliakis A, Mortaki D, Zacharatou A, et al. Eyebrow involvement in frontal fibrosing alopecia: a clinicopathologic cohort study for the reversibility of hair loss. J Am Acad Dermatol. 2020;82(3):755-7.

7 Anzai A, Donati A, Valente NY, Romiti R, Tosti A. Isolated eyebrow loss in frontal fibrosing alopecia: relevance of early diagnosis and treatment. Br J Dermatol. 2016;175(5): 1099-101.

8 Pekmezci E, Turkoğlu M, Gökalp H, Kutlubay Z. Minoxidil downregulates interleukin-1 alpha gene expression in $\mathrm{HaCaT}$ cells. Int J Trichology. 2018;10(3):108-12. 